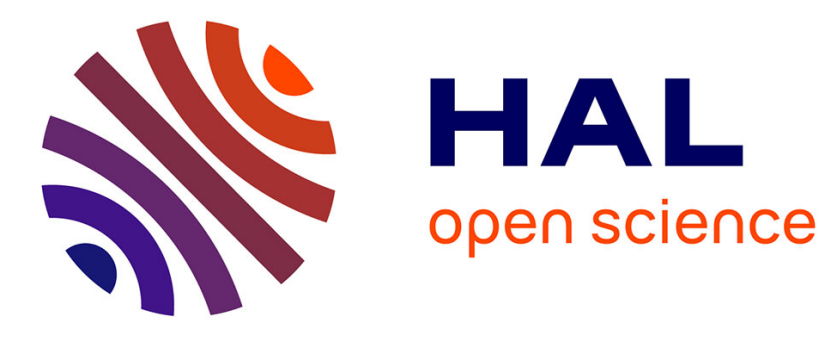

\title{
Réchauffement climatique et ressource neige en domaines skiables \\ Pierre Paccard
}

\section{To cite this version:}

Pierre Paccard. Réchauffement climatique et ressource neige en domaines skiables. Neige et glace de montagne: Reconstitution, dynamique, pratiques, 8, Collection EDYTEM - Cahiers de Géographie, nº, pp.181-192, 2009. halsde-00387306

\section{HAL Id: halsde-00387306 https://hal.science/halsde-00387306}

Submitted on 1 Apr 2011

HAL is a multi-disciplinary open access archive for the deposit and dissemination of scientific research documents, whether they are published or not. The documents may come from teaching and research institutions in France or abroad, or from public or private research centers.
L'archive ouverte pluridisciplinaire HAL, est destinée au dépôt et à la diffusion de documents scientifiques de niveau recherche, publiés ou non, émanant des établissements d'enseignement et de recherche français ou étrangers, des laboratoires publics ou privés. 


\section{Collection EDYTEM}

Numéro 8 - Année 2009

Cahiers de

Géographie

\section{NEIGE et GLACE de MONTAGNE}

Reconstitution, dynamique, pratiques

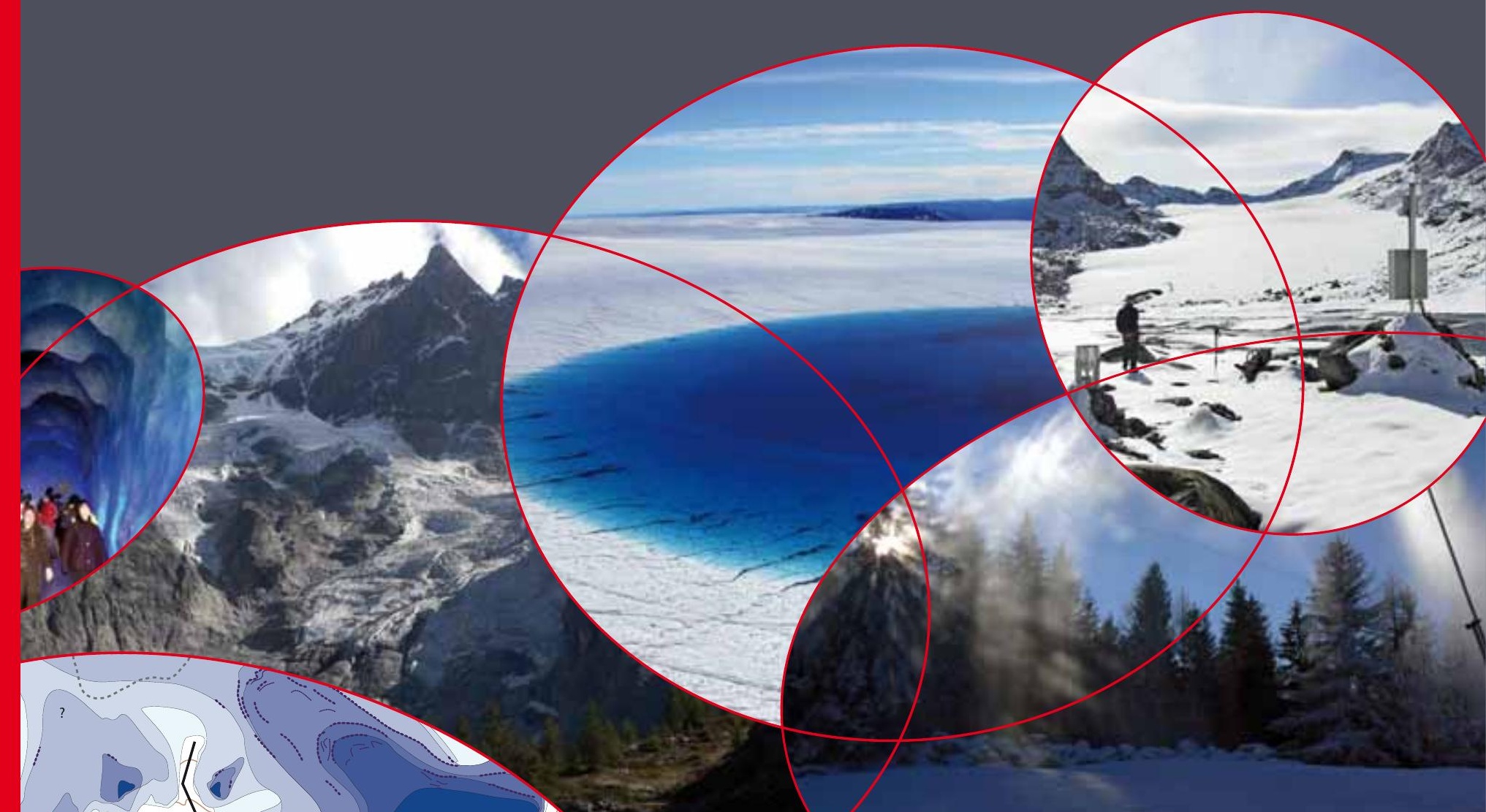





\title{
RECHAUFFEMENT CLIMATIQUE ET RESSOURCE NEIGE EN DOMAINES SKIABLES
}

\author{
CLIMATE CHANGE AND SNOW RESSOURCE IN SKI RESORTS
}

\author{
PIERRE PACCARD \\ Laboratoire EDYTEM, Université de Savoie/CNRS, Campus scientifique, F 73376 Le Bourget-du-Lac cedex. \\ Contact : pierre.paccard@univ-savoie.fr
}

\begin{abstract}
RÉSUMÉ
Si la variabilité interannuelle des précipitations neigeuses est une réalité, la tendance à la diminution des quantités de neige en montagne est aujourd'hui un fait constaté et modélisé dans le cadre des perspectives du réchauffement climatique. La neige restant la principale ressource de l'activité des stations de sports d'hiver, les conséquences d'une diminution des quantités disponibles sur les domaines skiables ont d'ores et déjà été envisagées dans de nombreuses études. Reprenant et complétant une méthodologie existante, nous proposons ici une réflexion pour une évaluation de la fiabilité de l'enneigement naturel des stations de sports d'hiver à différents horizons temporels. Nous nous appuierons à la fois sur des données météorologiques et des informations relatives aux remontées mécaniques des stations étudiées. La méthodologie proposée est ici appliquée aux départements de Savoie et de HauteSavoie des Alpes françaises, départements comptant de nombreuses stations de sports d'hiver (ce travail s'inscrit dans le cadre d'un monitorat en entreprise réalisé au sein de la Direction Départementale de l'Equipement et de l'Agriculture de la Savoie). Au terme de cet exercice, l'analyse critique des résultats présentés permettra de relativiser ces derniers, au regard notamment de leur part d'incertitude. Notre propos restant centré sur une analyse du risque par l'aléa, il est enfin envisagé de compléter celui-ci par des recherches sur la prise en compte de ce risque par les stations de sports d'hiver et, en ce sens, sur leur capacité d'adaptation.
\end{abstract}

MotS-CLÉS : STATIONS DE SPORTS D'HIVER, RÉCHAUFFEMENT CLIMATIQUE, RESSOURCE NEIGE, FIABILITÉ DE L'ENNEIGEMENT, RÉFLEXION MÉTHODOLOGIQUe, SAVOIE ET HAUTE-SAVOIE.

\begin{abstract}
If the interannual variability of the abundance or the rareness of snowfalls is a reality, the declining trend in mountain snow quantity is today an observed fact but also envisaged by numerous modelisations in the current and future context of warmer temperatures. The snow being the principal resource of the ski resorts activity, consequences of a decrease in the quantities available on the ski area have already been considered by several works. By reusing and supplementing an existing methodology, we propose here a reflection for the evaluation of the ski resorts natural snow coverage reliability at different time horizons, based on both weather data and information related to the lifts of the resorts studied. The proposed methodology is here applied to the Savoie and HauteSavoie departments of the French Alps, which both contain many ski resorts (this work comes within the framework of a company tutoring realised within the Savoie's Departmental Direction of Equipment and Agriculture). At the end of this exercise, the critical analysis of the presented results will allow us to relativize these ones, particularly regarding to their uncertainty. Our aim being based on the risk analysis of the randomization, it is finally considered to complete it by researches on the consideration of this risk by the ski resorts and, by that, on their capacity of adapation.
\end{abstract}

KEYWORDS: SKI RESORTS, GLOBAL WARMING, SNOW RESOURCE, SNOW-RELIABILITY, METHODOLOGICAL REFLECTION, SAVOIE AND HAUTE-SAVOIE. 


\section{INTRODUCTION}

Il est un paradoxe certain entre le sujet proposé et l'abondance de neige de l'hiver 2008-2009 qui nous invite avant toute chose à relativiser notre propos. Le 5 février 2009, Météo France établissait ainsi en ces termes un point sur l'enneigement dans les massifs français (Météo France, 2009) : « en ce début de février, tous les massifs montagneux français bénéficient d'un bon enneigement. Il est même particulièrement remarquable en Corse et dans les massifs situés les plus au sud des Alpes » (photo 1). A titre d'exemple, une telle abondance de neige n'avait plus été connue dans le Queyras depuis l'hiver 1978-1979. Encore plus au sud, la route menant à la station d'Isola 2000 est restée fermée pendant plusieurs jours au début du mois de février 2009 en raison d'un fort risque d'avalanche, tant les précipitations neigeuses furent importantes.

Si la variabilité climatique interannuelle est dès lors certaine, celle-ci ne doit cependant pas être confondue avec la variation du climat sur le long terme. Nous proposons dans ce cadre une réflexion sur les interactions possibles entre le changement climatique actuel et la ressource neige en stations de sports d'hiver. Ainsi, dans une démarche prospective et constructive, après avoir fait un point sur l'état de l'art, nous essaierons de compléter l'une des approches présentées, puis de l'appliquer aux stations de sports d'hiver des départements français alpins de Savoie et de Haute-Savoie. Cet exercice bénéficiera par ailleurs d'une large analyse critique.

Au titre de cette contribution, nous ne prétendons évidemment pas apporter des résultats formels, tant les incertitudes sont grandes. Nous questionnerons plutôt les différentes analyses possibles, tant en termes de données à exploiter que de méthodologies à conduire.

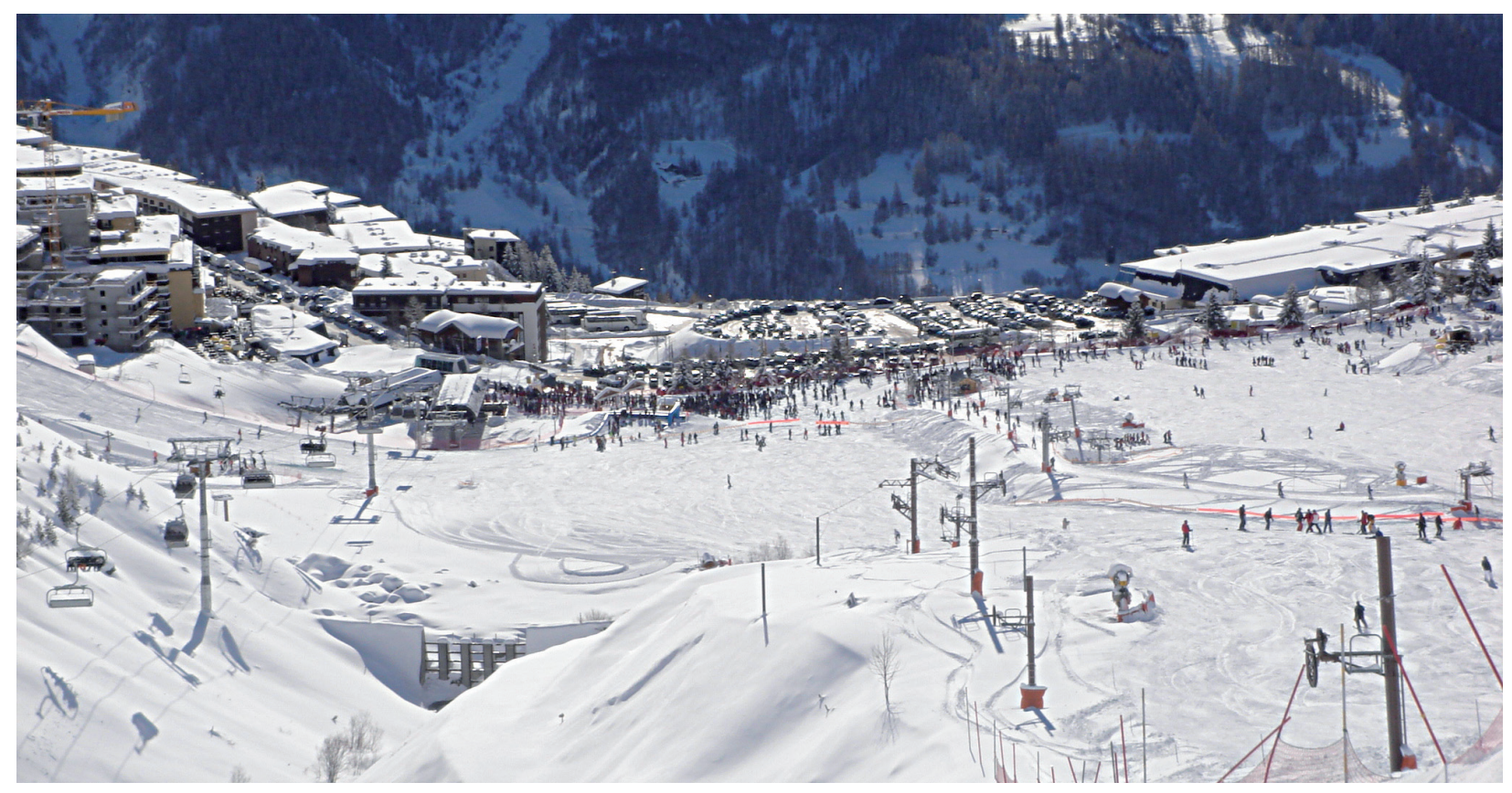

Photo 1 - Le bon enneigement de l'hiver 2008-2009 dans les Alpes du Sud. Ici le front de neige de la station d'Orcières (Hautes Alpes) au mois de janvier 2009.

\section{I - ETAT DE L'ART}

Depuis plusieurs années, l'étude de séries météorologiques alpines tend à montrer une diminution du nombre de jours de neige au sol par an (Etchevers et Martin, 2002 ; Laternser et Schneebeli, 2003). Dans le cadre du réchauffement climatique actuel, ces diminutions pourraient être expliquées par une fonte plus précoce du manteau neigeux, du fait de températures plus douces, ainsi que par une possible élévation altitudinale de la limite pluie/neige (Etchevers et Martin, 2002 ; Aspen Global Change Institute, 2006 ; PrudentRichard, 2008). La neige étant le principal support de l'activité des stations de sport d'hiver, elle constitue une ressource indispensable à leur fonctionnement. C'est pourquoi, au vu de cet enjeu, les conséquences d'une diminution de l'enneigement naturel ont d'ores et déjà fait l'objet de nombreuses études. Si certaines méthodologies visent plutôt à étudier de façon rétrospective comment les stations de sports d'hiver ont déjà réagi à de précédents hivers peu généreux en neige, d'autres, dans une démarche prospective, tentent d'évaluer leurs probabilités d'enneigement pour les prochaines années. 


\section{1 - L'analyse de la variation des chiffres d'affaires}

Les différentes sensibilités des stations aux « mauvais » hivers passés sont généralement présentées par l'étude de la variation de leurs chiffres d'affaires au cours du temps. L'idée est ici qu'une station sensible à l'aléa d'enneigement connaîtra de fortes variations de son chiffre d'affaires entre de «bons » et de « mauvais » hivers. Au contraire, le chiffre d'affaires d'une station relativement protégée des déficits d'enneigement, comme une station située en haute altitude par exemple, se présentera comme plutôt stable dans le temps. Il est ainsi possible que «la résistance des stations aux mauvais hivers passés nous renseigne sur leur capacité à faire face au changement climatique annoncé » (Berlioz, 2008). Cependant, pouvoir faire face aux fluctuations du climat actuel ne signifie $a$ priori pas que l'on peut faire face à des fluctuations d'un climat plus chaud.

Ce type d'approche est proposé par ODIT France (2008) qui évalue la sensibilité des exploitations de remontées mécaniques aux aléas conjoncturels à partir de l'amplitude moyenne des variations saisonnières de chiffre d'affaires sur la période 1990-2007 (figure 1). En employant cette méthode sur l'ensemble du parc de domaines skiables de chaque massif français, ODIT France note ainsi « de fortes disparités d'un massif à l'autre qui rendent certains territoires particulièrement vulnérables, ainsi que des disparités locales parfois importantes au sein de chacun des massifs »(ODIT France, 2008). A titre d'exemple, la majeure partie des

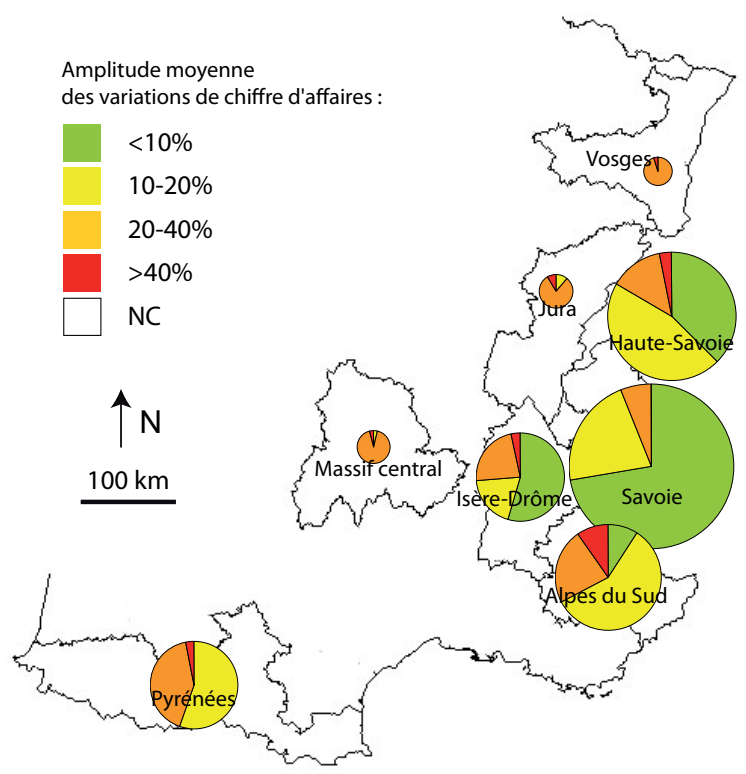

Figure 1 - Amplitude moyenne des variations saisonnières de chiffre d'affaires des exploitations de remontées mécaniques sur la période 1990-2007 (ODIT France, modifié, 2008). La taille des secteurs est proportionnelle à la moyenne du chiffre d'affaires dégagé par saison. exploitations de remontées mécaniques du département de la Savoie connait des amplitudes de variation de son chiffre d'affaires inférieures à $10 \%$ tandis que ces amplitudes sont comprises entre 10 et $20 \%$ pour la plus grande partie des exploitations de Haute-Savoie.

Pour ce qui est des disparités locales à l'intérieur d'un même massif, on peut citer l'exemple de la Savoie pour qui, selon ces mêmes principes d'évaluation, «les stations les plus menacées se concentrent dans le Val d'Arly et les Bauges, et ne représentent qu'une faible partie de l'offre ski »(Berlioz, 2008). Des variations de chiffre d'affaires comprises entre 20 et $40 \%$ sont en effet connues pour certaines des stations de ces massifs.

Il est à noter qu'une telle analyse ne reflète pas seulement la variabilité de l'enneigement d'un domaine skiable mais également d'autres paramètres, tels que l'origine des clientèles. ODIT France parle ainsi de sensibilité aux aléas conjoncturels et non de sensibilité à la seule variabilité de l'enneigement. Cette idée est partagée par Hélion (2006) qui explique, après avoir comparé les variations de chiffres d'affaires de l'ensemble des stations des Alpes françaises entre les saisons 1991-1992 et 1992-1993 (la saison 1992-1993 étant une saison mal enneigée), que « les plus grosses pertes correspondent aux stations proches des grandes aires urbaines : ce sont celles qui sont marquées par le ski des populations locales » (Hélion, 2006).

\section{2 - Les modèles prospectifs}

En réponse à ces premières méthodes d'évaluation de la résistance des stations aux mauvais hivers passés, une autre approche consiste à envisager les probabilités futures d'enneigement des sites considérés. En France, sur la base des projections des modèles climatiques de Météo France, Tabeaud et Delaporte (2005) identifient quatre groupes de stations pour lesquelles «les menaces pour le futur sont différentes ». Le premier groupe correspond aux stations qui n'ont pas de problème majeur, c'est-à-dire les grandes stations dotées de domaine de haute altitude, où les problèmes d'enneigement sont contenus. Le second groupe est constitué par les stations où le risque est occasionnel, en moyenne montagne, plus dépendantes de l'enneigement mais rattachées à un domaine de haute altitude. En troisième lieu viennent les stations où le risque sera fréquent à l'avenir, c'est-à-dire les stations de moyenne altitude sans domaine de haute altitude. Enfin, les stations de sports d'hiver où la reconversion est nécessaire, sont essentiellement des stations de sports d'hiver de première génération touchées directement par la rareté de la neige en dessous de $1000 \mathrm{~m}$ (Tabeaud et Delaporte, 2005).

Loubier (2007) propose par ailleurs une démarche de simulation climatique à l'horizon 2015 appliquée aux domaines skiables de Savoie et Haute-Savoie. En 
établissant des «cartes fondées sur la représentation d'une probabilité de survenue de neige en cas de précipitation » (Loubier, 2007), celui-ci estime que la probabilité d'une faible couverture neigeuse se fera sentir plus tard en début de saison et plus tôt en fin de saison. Le changement climatique pouvant ainsi fortement affecter l'économie du ski.

Aux Etats-Unis, une étude publiée en 2006 par l'Aspen Global Change Institute simule, sur la base de projections établies par des modèles climatiques, les conséquences du réchauffement climatique sur la station d'Aspen à l'horizon 2100 (Aspen est une station de ski du Colorado, dans les Montagnes Rocheuses à l'ouest des Etats-Unis). L'une des conclusions de cette projection indique qu'à l'avenir, les précipitations tomberaient à Aspen d'avantage sous la forme de pluie que de neige et que, si l'augmentation des émissions de gaz à effet de serre ne cesse pas, la pratique du ski serait fortement compromise à l'horizon 2100. La réduction des émissions pourrait néanmoins préserver la pratique du ski aux altitudes moyennes et supérieures (Aspen
Global Change Institute, 2006).

Au Canada, les travaux de Dawson (2008) sur les stations de sports d'hiver canadiennes et américaines tendent à montrer une érosion de l'offre de ski proposée, tant par la diminution déjà constatée du nombre de domaines skiables (plus de 700 en 1984 à moins de 500 en 2006 sur l'ensemble du territoire des Etats-Unis) que par les modélisations réalisées dans la perspective du réchauffement climatique. En revanche, les enquêtes de clientèles conduites montrent que les évolutions possibles des pratiques de la clientèle face à de potentiels manques de neige en stations restent aujourd'hui incertaines (Dawson, 2008).

Enfin, les travaux de l'OCDE (2007) se sont précisément attachés à définir, sur l'ensemble des pays de l'arc alpin, le nombre de stations de sports d'hiver considérées comme fiables du point de vue de l'enneigement naturel à l'heure actuelle et dans la perspective du réchauffement climatique. Cette approche fait l'objet d'une présentation plus détaillée dans le paragraphe suivant.

\section{II - L'APPROCHE PROSPECTIVE PROPOSÉE PAR L'OCDE (2007) LE CONCEPT DE LIMITE DE FIABILITÉ DE L'ENNEIGEMENT NATUREL}

Les projections réalisées par l'OCDE reposent sur deux hypothèses principales. La première, communément admise, est que «pour exploiter un domaine skiable avec un résultat satisfaisant, il faut un manteau neigeux suffisant pour la pratique du ski pendant au moins cent jours par saison 》 avec une épaisseur de neige minimum au sol de 30 centimètres (OCDE, 2007). Cette hypothèse peut être confrontée au nombre moyen de jours d'ouverture sur l'ensemble des stations françaises au cours d'une saison: 101 jours d'ouverture à titre d'exemple pour l'hiver 2007-2008 considéré comme plutôt bien enneigé. Pour la même saison, cette durée était de 124 jours pour les 100 stations françaises au plus grand chiffre d'affaires et respectivement 126 jours et 130 jours pour les principales stations de Haute-Savoie et de Savoie (Montagne Leader, 2008).

A partir de cette première hypothèse, en extrapolant des résultats de travaux réalisés pour la Suisse (Föhn, 1990 ; Laternser et Schneebeli,
2003), une limite de fiabilité de l'enneigement naturel est définie par l'OCDE pour chacune des zones alpines prises en compte dans l'analyse. Cette altitude - c'est à dire l'altitude minimale où l'on retrouverait au moins 100 jours de neige avec $30 \mathrm{~cm}$ de neige au sol par saison - a été définie à 1200 mètres pour l'ensemble des départements français des Alpes du Nord : Isère, Savoie et Haute-Savoie (Tableau 1). En outre, cette limite altitu-

\begin{tabular}{|c|c|c|c|c|c|}
\hline $\begin{array}{c}\text { Limite de la } \\
\text { fiabilité de } \\
\text { l'enneige- } \\
\text { ment naturel }\end{array}$ & France & Suisse & Autriche & Italie & Allemagne \\
\hline $1050 \mathrm{~m}$ & & & $\begin{array}{l}\text { - Pays de Salzbourg } \\
\text { - Styrie } \\
\text { - Haute-Autriche } \\
\text { - Basse-Autriche }\end{array}$ & & - Haute-Bavière \\
\hline & - Isère & \multirow[b]{2}{*}{$\begin{array}{l}\text { - Alpes Vaudoises } \\
\text { et fribourgeoises } \\
\text { - Valais } \\
\text { - Oberland bernois } \\
\text { - Suisse centrale } \\
\text { - Suisse orientale } \\
\text { - Grisons }\end{array}$} & \multirow[b]{2}{*}{$\begin{array}{l}\text { - Vorarlberg } \\
\text { - Tyrol } \\
\text { - Carinthie (si l'on } \\
\text { considère que l'effet } \\
\text { «positif » de la } \\
\text { continentalité est } \\
\text { compensé par l'effet } \\
\text { «négatif » du } \\
\text { caractère méridional) } \\
\end{array}$} & & \multirow[t]{2}{*}{ - Souabe } \\
\hline $1200 \mathrm{~m}$ & $\begin{array}{l}\cdot \text { Savoie } \\
\cdot \text { Haute-Savoie }\end{array}$ & & & & \\
\hline $1500 \mathrm{~m}$ & $\begin{array}{l}\cdot \text { Drôme } \\
\cdot \text { Hautes-Alpes } \\
\cdot \text { Alpes-de- } \\
\text { Haute-Provence } \\
\cdot \text { Alpes- } \\
\text { Maritimes }\end{array}$ & - Tessin & & $\begin{array}{l}\cdot \text { Piémont } \\
\cdot \text { Lombardie } \\
\cdot \text { Haut-Adige } \\
\text { - Frioul-Vénétie- } \\
\text { Julienne } \\
\text { - Trente }\end{array}$ & \\
\hline
\end{tabular}

Tableau 1 - Limite de fiabilité de l'enneigement naturel dans les zones alpines pour 100 jours de neige par an avec $30 \mathrm{~cm}$ de neige au sol (OCDE, 2007). Encadrée en rouge, la limite de fiabilité pour les départements de Savoie et Haute-Savoie : 1200 mètres. 
dinale remonterait de 150 mètres par degré de réchauffement moyen dans l'hypothèse de l'élévation des températures moyennes. Ainsi, dans l'hypothèse d'un réchauffement de $1{ }^{\circ} \mathrm{C}, 2^{\circ} \mathrm{C}$ ou $4^{\circ} \mathrm{C}$ des températures moyennes (scénarios retenus dans l'analyse proposée par l'OCDE), cette altitude passerait de 1200 mètres en Savoie et Haute-Savoie à respectivement 1350 mètres, 1600 mètres ou 1800 mètres.

Enfin, selon l'OCDE - il s'agit là de la seconde hypothèse pour la projection proposée -, « un domaine skiable donné est considéré comme fiable du point de vue de son enneigement naturel si la moitié supérieure de la plage d'altitude dans laquelle il se situe se trouve au-dessus de la valeur seuil de la limite de la fiabilité de l'enneigement naturel »(OCDE, 2007). La plage d'altitude considérée correspond en fait à l'espace situé entre le point haut (le sommet) et le point bas (le pied des pistes) d'un domaine skiable.

Les résultats de cette projection ont été obtenus en confrontant les altitudes de fiabilité précédemment

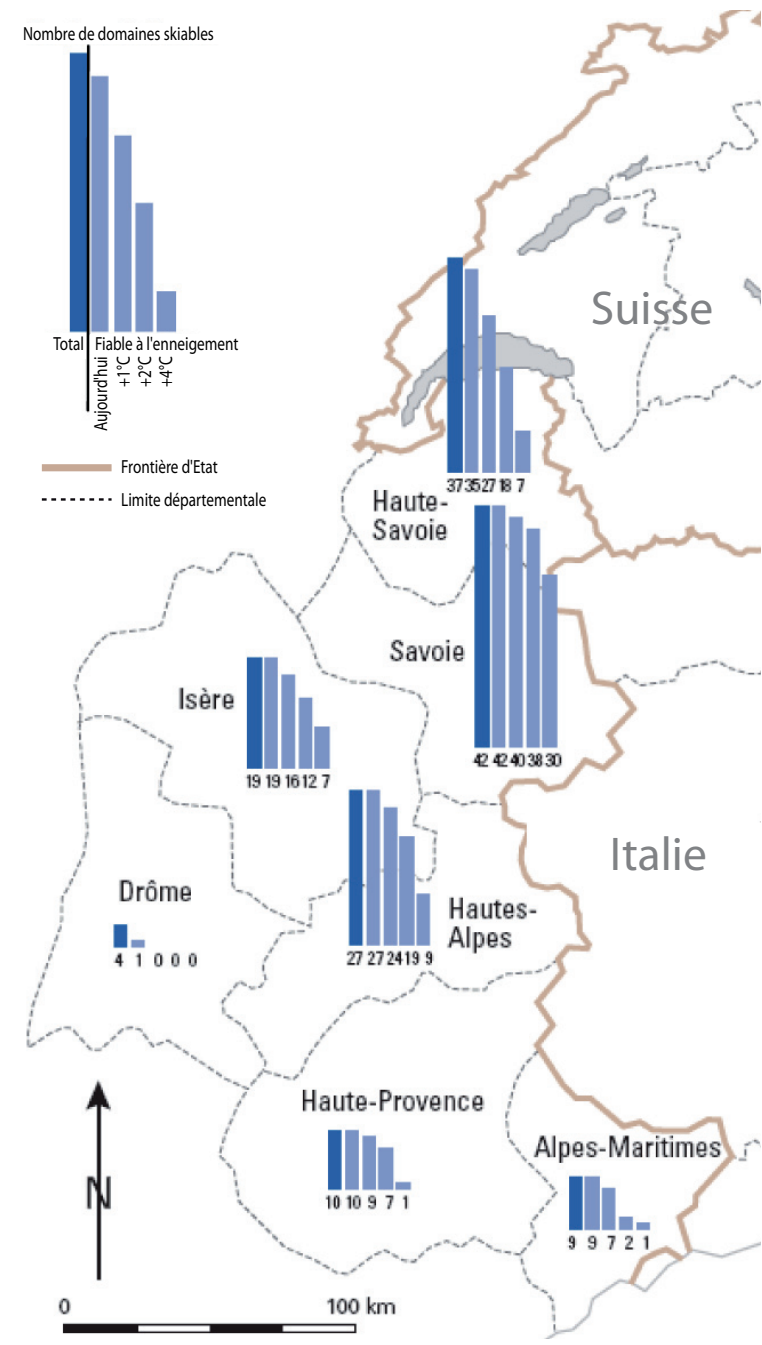

Figure 2 - Nombre de domaines skiables offrant un enneigement naturel fiable dans les Alpes françaises, aujourd'hui et dans les conditions climatiques de demain (OCDE, modifié, 2007). définies avec les plages d'altitudes des domaines skiables alpins (figure 2). En Savoie, l'OCDE conclut qu'un recul de $300 \mathrm{~m}$ de la limite de la fiabilité de l'enneigement naturel $\left(2^{\circ} \mathrm{C}\right.$ supplémentaires d'ici 2050) ramènerait le nombre de domaines skiables disposant d'un enneigement naturel fiable à $90 \%$ environ du total actuel (ibid.). Les résultats pour le département de la Haute-Savoie, dont les domaines skiables se trouvent à des altitudes moins élevées, c'est-à-dire principalement situés en moyenne montagne, sont plus sévères : $49 \%$ seulement des domaines skiables du département seraient considérés comme toujours fiables pour un réchauffement de $2^{\circ} \mathrm{C}$.

Les résultats présentés par l'OCDE offrent la possibilité de pouvoir comparer, de façon relative, le degré de sensibilité au réchauffement climatique du parc de domaine skiable de chaque secteur étudié. Cependant, l'analyse proposée par l'OCDE, bien que remarquable en termes de superficie couverte - l'ensemble des Alpes européennes -, présente à notre sens 3 principales limites.

Tout d'abord, une limite de fiabilité est définie à l'échelle de plusieurs départements sans prendre en compte les différences de climat entre les massifs. Ces différences climatiques, en termes de température, de précipitation (solide ou liquide), sont pourtant grandes entre les massifs des départements pour lesquels une même altitude de fiabilité a été définie. A titre d'exemple, le climat de la Haute Maurienne assez sec (vallée intra-alpine du département de la Savoie) contraste avec les précipitations abondantes que l'on peut retrouver sur l'ensemble des massifs préalpins des départements savoyards : Chablais, Bauges et Chartreuse.

Les plages d'altitude des domaines skiables sont ensuite réduites à une altitude moyenne. Si l'altitude d'un domaine skiable peut effectivement être définie par la moyenne altitudinale entre le point haut et le point bas d'un domaine, la plus grande capacité de production de ski peut en réalité se trouver soit à haute altitude, et s'affranchir en ce sens du risque d'un faible enneigement à basse altitude, soit à basse altitude, et être en ce sens relativement vulnérable à un risque de faible enneigement. Cette capacité de production de ski peut se mesurer par le moment de puissance d'une installation ou d'une station. Celui-ci se définit par le produit de son débit et de sa dénivelée (ODIT France, 2006).

Finalement, des résultats sont présentés en nombre de sites impactés, sans prise en compte de la place qu'occupe chacun d'entre eux dans l'offre touristique globale. En effet, il est une grande différence entre un stade de neige périurbain et une grande station d'altitude dans le poids qu'ils occupent en matière d'offre de ski. Une analyse site par site, ou par exemple en nombre de journées skieurs (Reynaud, 2008), rétablirait ainsi ces différences inter-station qu'une analyse globale, sur l'ensemble d'un parc de domaines skiables, tend au contraire à lisser. 
En réalité, les principales lacunes de la projection proposée par l'OCDE peuvent être liés à l'échelle de travail retenue. Une analyse à l'échelle de l'ensemble des Alpes européennes ne peut en effet approcher le même degré de précision qu'une analyse à une échelle plus fine, celle par exemple de deux départements comme on se propose de le faire. En reprenant les bases des travaux de l'OCDE, notre contribution va désormais s'attacher à essayer de palier les principales limites ci-dessus explicitées en tentant d'affiner l'approche puis de l'appliquer aux départements alpins français de Savoie et de Haute-Savoie. Ce travail est le fruit d'une réflexion conduite conjointement avec les services de l'Etat des départements de Savoie et Haute-Savoie (Directions Départementales de l'Equipement et de l'Agriculture), de la région Rhône-Alpes (Direction Régionale de l'Environnement) et ODIT France dans le cadre d'un monitorat en entreprise. Ces résultats préliminaires seront prochainement complétés pour faire l'objet d'un rapport à part entière.

\section{III - REPRISE DES TRAVAUX DE L'OCDE application auX départements de Savoie et Haute-Savoie}

\section{1 - Quelles altitudes de fiabilité de l'enneigement naturel pour les départements de Savoie et Haute-Savoie?}

L'exercice consiste dans un premier temps à essayer de régionaliser par massif la limite de fiabilité de l'enneigement naturel définie à $1200 \mathrm{~m}$ par l'OCDE pour l'ensemble des départements de Savoie et HauteSavoie. Dans un premier temps, les résultats des modèles SAFRAN et CROCUS de Météo France (Etchevers et Martin, 2002) nous permettent de mettre en évidence les différences d'enneigement selon les massifs considérés, notamment les durées d'enneigement plus impor-

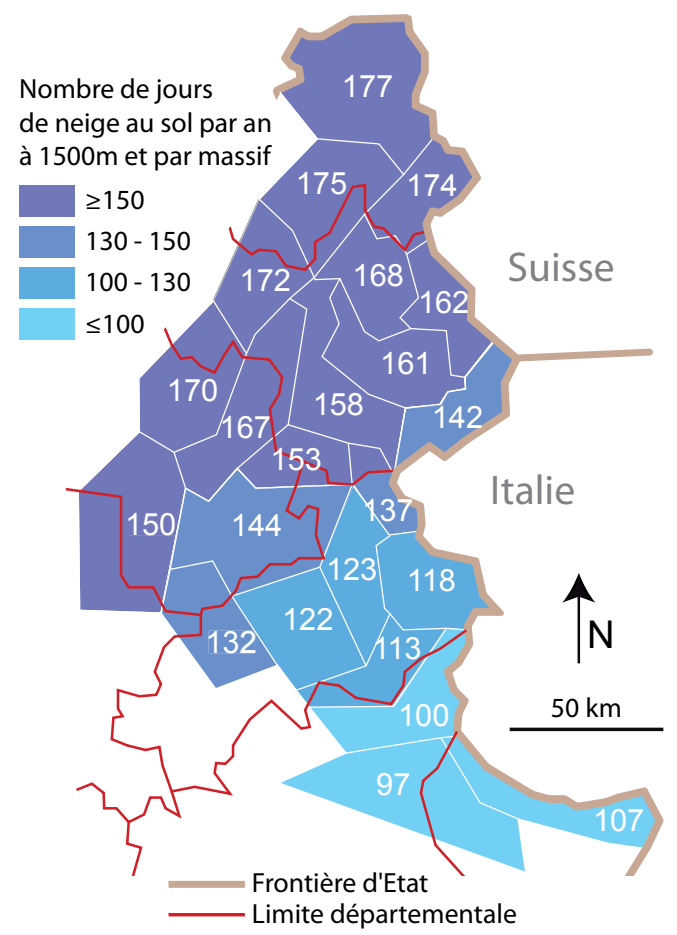

Figure 3 - Durée moyenne de l'enneigement à $1500 \mathrm{~m}$ (en jours par an), simulée par SAFRAN et CROCUS dans les Alpes en 2002 (Etchevers et Martin, 2002, in ODIT France, modifé, 2006). tantes pour les massifs préalpins (figure 3). En Savoie et Haute-Savoie, le nombre de jours avec de la neige au sol simulé à 1500 mètres (dans les conditions climatiques de 2002) varie selon un gradient Nord-Ouest / Sud-Est, de 177 jours dans le massif du Chablais à 142 jours dans la vallée de la Haute Maurienne.

A partir des résultats de cette modélisation, il est possible de pondérer, d'une façon très simple et très théorique, l'altitude de fiabilité de l'enneigement naturel proposée à 1200 mètres par l'OCDE pour l'ensemble des départements de Savoie et Haute-Savoie. Les résultats et le calcul de cette pondération sont présentés dans le tableau 2.

Dans un second temps, ces résultats obtenus en première approximation doivent être confrontés aux relevés Météo France des hauteurs de neige quotidienne au

\begin{tabular}{|c|c|c|}
\hline Massif & $\begin{array}{l}\text { Nombre de } \\
\text { jours de } \\
\text { neige au sol } \\
\text { à } 1500 m^{[1]} \\
N_{\text {j neige } 1500}\end{array}$ & $\begin{array}{l}\text { Limite de } \\
\text { fiabilité } \\
\text { pondérée }{ }^{[2]} \\
\text { (en mètres) } \\
\text { Alti }_{\text {fiab massif }}\end{array}$ \\
\hline Chablais & 177 & 1134 \\
\hline Aravis & 175 & 1146 \\
\hline Mont Blanc & 174 & 1152 \\
\hline Bauges & 172 & 1164 \\
\hline Haute Tarentaise & 162 & 1224 \\
\hline Beaufortin Val d'Arly & 168 & 1188 \\
\hline Vanoise & 161 & 1230 \\
\hline Haute Maurienne & 142 & 1345 \\
\hline Belledonne & 167 & 1194 \\
\hline Maurienne & 158 & 1248 \\
\hline Chartreuse & 170 & 1176 \\
\hline Moyenne (Savoie, Haute-Savoie) & 163 & $1200[3]$ \\
\hline
\end{tabular}

Tableau 2 - Limite de fiabilité de l'enneigement naturel pondérée par massif. Les résultats de calcul figurent en italique dans ce tableau.

${ }^{[1]}$ Donnée du modèle SAFRAN et CROCUS de Météo France ;

${ }^{[2]}$ Alti $_{\text {fiab massif }}=1200 \times\left(365-N_{\text {jneige } 1500}\right) /$ Moyenne de $N_{\text {jneige } 1500}$;

[3] Donnée OCDE, 2007. 
sol. En effet, à partir de ces mesures et pour chaque station météorologique considérée, le nombre de jours avec une hauteur de neige au sol supérieure à $30 \mathrm{~cm}$ peut être connu.

Les hauteurs de neige quotidiennes au sol de 10 stations météorologiques Météo France situées en Haute-Savoie et de 18 stations situées en Savoie ont ainsi été traitées (figure 4). Elles ont permis de connaître le nombre de jours moyen par hiver avec plus de $30 \mathrm{~cm}$ de neige au sol. Ces données n'ont malheureusement pu être analysées que pour les quatre hivers suivant l'année 2004, les hauteurs de neige au sol n'étant connu avant cette date pour aucune des stations météorologiques étudiées. Les résultats sont présentés dans le tableau 3 en considérant ou non l'hiver 20062007 particulièrement peu enneigé et illustrés en figure 5 sans considérer ce même hiver.

Figure 4 - Localisation des stations météorologiques Météo France dont les données de hauteur de neige ont été étudiées.

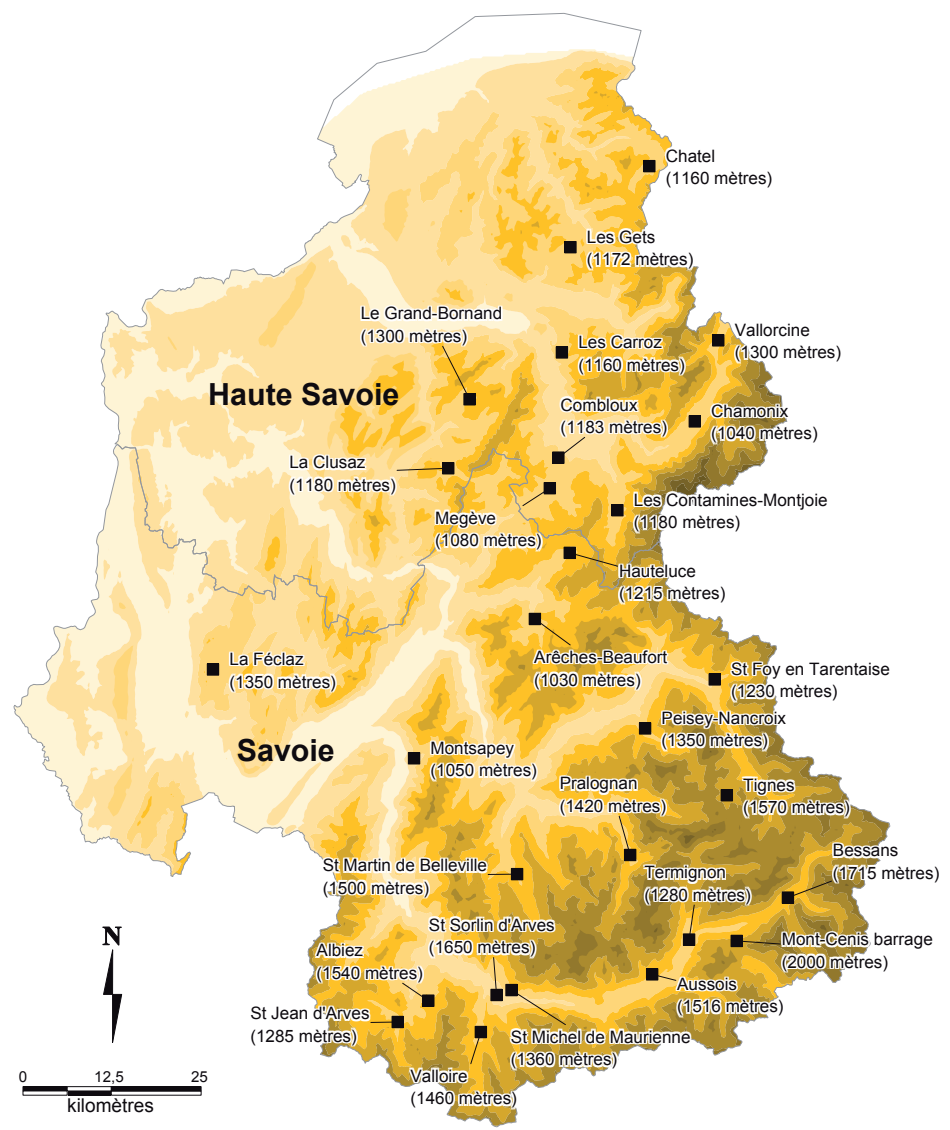

\begin{tabular}{|c|c|c|c|}
\hline Station & Massif & Altitude & $\begin{array}{l}\text { Nombre moyen de jours par hiver } \\
\text { avec } 30 \mathrm{~cm} \text { de neige au sol } \\
\text { (entre parenthèses, avec } 2006-2007 \text { ) }\end{array}$ \\
\hline Chatel & Chablais & 1160 & $96(72)$ \\
\hline Les Carroz & Chablais & 1160 & $76(57)$ \\
\hline Les Gets & Chablais & 1172 & $83(64)$ \\
\hline Chamonix & Mont Blanc & 1040 & $39(30)$ \\
\hline Les Contamines-Montjoie & Mont Blanc & 1180 & $59(46)$ \\
\hline Vallorcine ${ }^{["]}$ & Mont Blanc & 1300 & 96 \\
\hline Combloux & Aravis & 1183 & $71(54)$ \\
\hline La Clusaz & Aravis & 1180 & $104(73)$ \\
\hline Le Grand-Bornand & Aravis & 1300 & $113(96)$ \\
\hline Megève & Beaufortain - Val d'Arly (74) & 1080 & $92(70)$ \\
\hline Arêches-Beaufort & Beaufortain - Val d'Arly & 1030 & $77(59)$ \\
\hline Hauteluce & Beaufortain - Val d'Arly & 1215 & $126(109)$ \\
\hline La Féclaz & Bauges & 1350 & $124(105)$ \\
\hline Pralognan & Tarentaise & 1420 & $126(114)$ \\
\hline St Martin de Belleville & Tarentaise & 1500 & $65(51)$ \\
\hline Peisey-Nancroix & Haute Tarentaise & 1350 & 34 (26) \\
\hline Ste Foy en Tarentaise ${ }^{[*]}$ & Haute Tarentaise & 1230 & $60(41)$ \\
\hline Tignes ${ }^{[*]}$ & Haute Tarentaise & 1570 & $134(94)$ \\
\hline Albiez & Maurienne & 1540 & $116(103)$ \\
\hline Montsapey & Maurienne & 1050 & $8(7)$ \\
\hline St Jean d'Arves & Maurienne & 1285 & $54(41)$ \\
\hline St Michel de Maurienne & Maurienne & 1360 & $31(24)$ \\
\hline St Sorlin d'Arves & Maurienne & 1650 & $121(98)$ \\
\hline Valloire & Maurienne & 1460 & $85(57)$ \\
\hline Aussois & Haute Maurienne & 1516 & $61(47)$ \\
\hline Bessans $\left[{ }^{\star}\right]$ & Haute Maurienne & 1715 & $131(108)$ \\
\hline Mont-Cenis barrage & Haute Maurienne & 2000 & $113(92)$ \\
\hline Termignon & Haute Maurienne & 1280 & $74(56)$ \\
\hline
\end{tabular}

Tableau 3 - Nombre moyen de jours par hiver avec $30 \mathrm{~cm}$ de neige au sol depuis l'hiver 2004-2005, avec ou sans considérer l'hiver 2006-2007. Données : Météo France ([*] Séries de données incomplètes ; moyenne uniquement sur : 2004-2005 pour Vallorcine ; 2004-2005, 2006-2007 et 2007-2008 pour Ste Foy Tarentaise ; 2006-2007 et 2007-2008 pour Tignes ; 2005-2006, $2006-2007$ et 2007-2008 pour Bessans) 


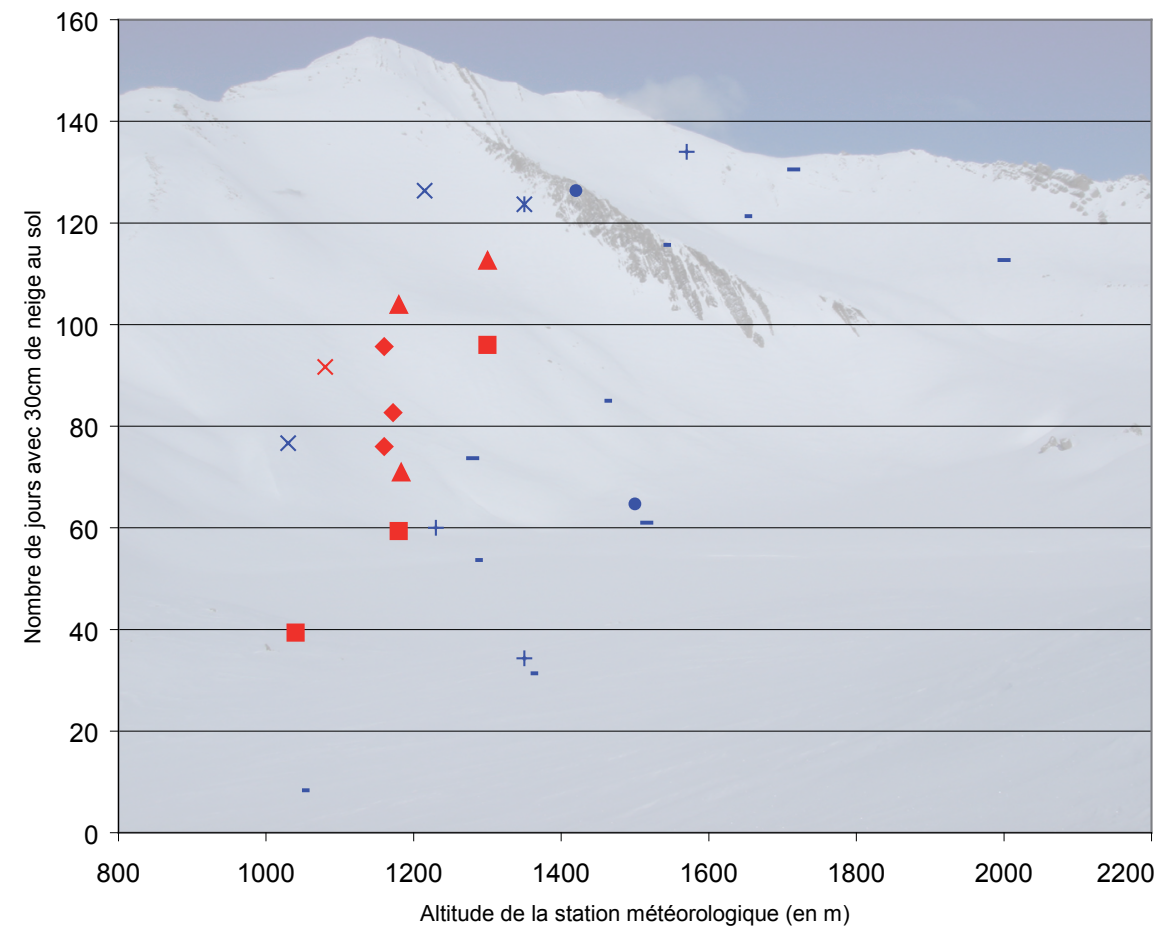

Stations météos, par massif (en rouge : Haute-Savoie, en bleu : Savoie)

$\checkmark$ Chablais

Mont Blanc

$\triangle$ Aravis

$\times$ Beaufortain - Val d'Arly (74)

$\times$ Beaufortain - Val d'Arly

* Bauges

- Tarentaise

+ Haute Tarentaise

- Maurienne

- Haute Maurienne

Figure 5 - Nombre moyen de jours par hiver avec $30 \mathrm{~cm}$ de neige au sol depuis l'hiver 2004-2005 en fonction de l'altitude de la station météorologique, sans considérer l'hiver 2006-2007. Les stations sont ici présentées selon leur appartenance aux différents massifs étudiés. Données : Météo France.
Les résultats ici présentés sont très hétérogènes. Il paraît difficile à la seule lecture du graphique de la figure 5 de pouvoir affecter par massif une altitude de fiabilité de l'enneigement, notamment du fait du nombre trop peu important de stations météorologiques étudiées et du peu de recul sur les années de mesures (seulement quatre hivers de données). Les 100 jours de neige sont atteints en Savoie pour des altitudes supérieures à $1400 \mathrm{~m}$, sauf pour une station du Beaufortain et une station des Bauges. Au contraire, la majorité des stations haut-savoyardes, toutes situées en dessous de 1400 mètres, n'atteignent pas les 100 jours de neige, exceptées deux stations du massif des Aravis.

Si la lecture des résultats obtenus en première approximation (Tableau 2) et l'analyse des données météorologiques permettent difficilement de statuer sur l'altitude de fiabilité de l'enneigement naturel sans équivoque, nous retiendrons tout de même pour la suite de notre raisonnement une altitude définie à :

- 1100 mètres pour l'ensemble des massifs préalpins (Chablais, Aravis, Bauges, Chartreuse, Aravis), du Beaufortain et du Mont-Blanc,

- 1200 mètres pour la Maurienne et la Tarentaise,

- 1300 mètres pour la Haute Tarentaise,

- 1400 mètres pour la Haute Maurienne.

Ce choix tient compte des précipitations plus importantes, à la même altitude, pour les massifs préalpins au regard des massifs plus internes.

Ces altitudes ainsi définies ont également été modulées selon les scénarios proposés par l'OCDE (2007) : augmentation de 150 mètres, 300 mètres et 600 mètres des altitudes de fiabilité de l'enneigement naturel pour un réchauffement respectif de $1^{\circ} \mathrm{C}, 2^{\circ} \mathrm{C}$ ou $4^{\circ} \mathrm{C}$. A titre d'exemple, si la fiabilité de l'enneigement naturel se situe actuellement à $1200 \mathrm{~m}$ pour un massif donné, elle se situerait à $1350 \mathrm{~m}, 1500 \mathrm{~m}$ ou $1800 \mathrm{~m}$ pour un réchauffement respectif de $1^{\circ} \mathrm{C}, 2^{\circ} \mathrm{C}$ ou $4^{\circ} \mathrm{C}$.

\section{2 - Quelles altitudes pour les domaines skiables de Savoie et Haute-Savoie?}

La base de données utilisée pour déterminer l'altitude des domaines skiables savoyards et haut-savoyards est le Fichier Informatisé des Remontées Mécaniques (FIRM) administré par les Directions Départementales de l'Equipement de Savoie et Haute-Savoie. La base de données FIRM recueille, par station de sports d'hiver (en l'occurrence par exploitant de remontées mécaniques), l'ensemble des remontées mécaniques existantes. Elle précise notamment l'altitude de départ, l'altitude d'arrivée et le débit skieur de chaque installation.

Grâce à cette base de données, les altitudes moyennes de chaque station de sports d'hiver ont pu être calculées selon trois méthodes différentes.

- méthode 1 : altitude moyenne définie par l'altitude du point le plus haut et du point le plus bas du domaine skiable ;

- méthode 2 : altitude moyenne définie par l'altitude de départ et d'arrivée de chaque remontée mécanique (moyenne des altitudes moyennes de chaque remontée mécanique) ;

- méthode 3 : altitude moyenne définie par l'altitude de départ et d'arrivée de chaque remontée mécanique pondérée par leur débit skieur (moyenne des altitudes moyennes de chaque remontée mécanique pondérées par leur débit skieur).

La principale motivation pour l'emploi de trois 
méthodes différentes pour ce raisonnement est le souhait de pouvoir recentrer l'altitude moyenne d'un domaine skiable en fonction du secteur où une station dispose de sa plus grande capacité de production de ski, c'est-à-dire le secteur où se trouve son plus grand moment de puissance.

\section{3 - Résultats et analyse critique}

Les altitudes de chaque station de sports d'hiver, calculées selon les trois méthodes explicitées, ont enfin été confrontées aux limites de fiabilité actuelles et futures de l'enneigement naturel définies pour chaque massif des départements de Savoie et Haute-Savoie. Les résultats sont présentés sur la figure 6 .

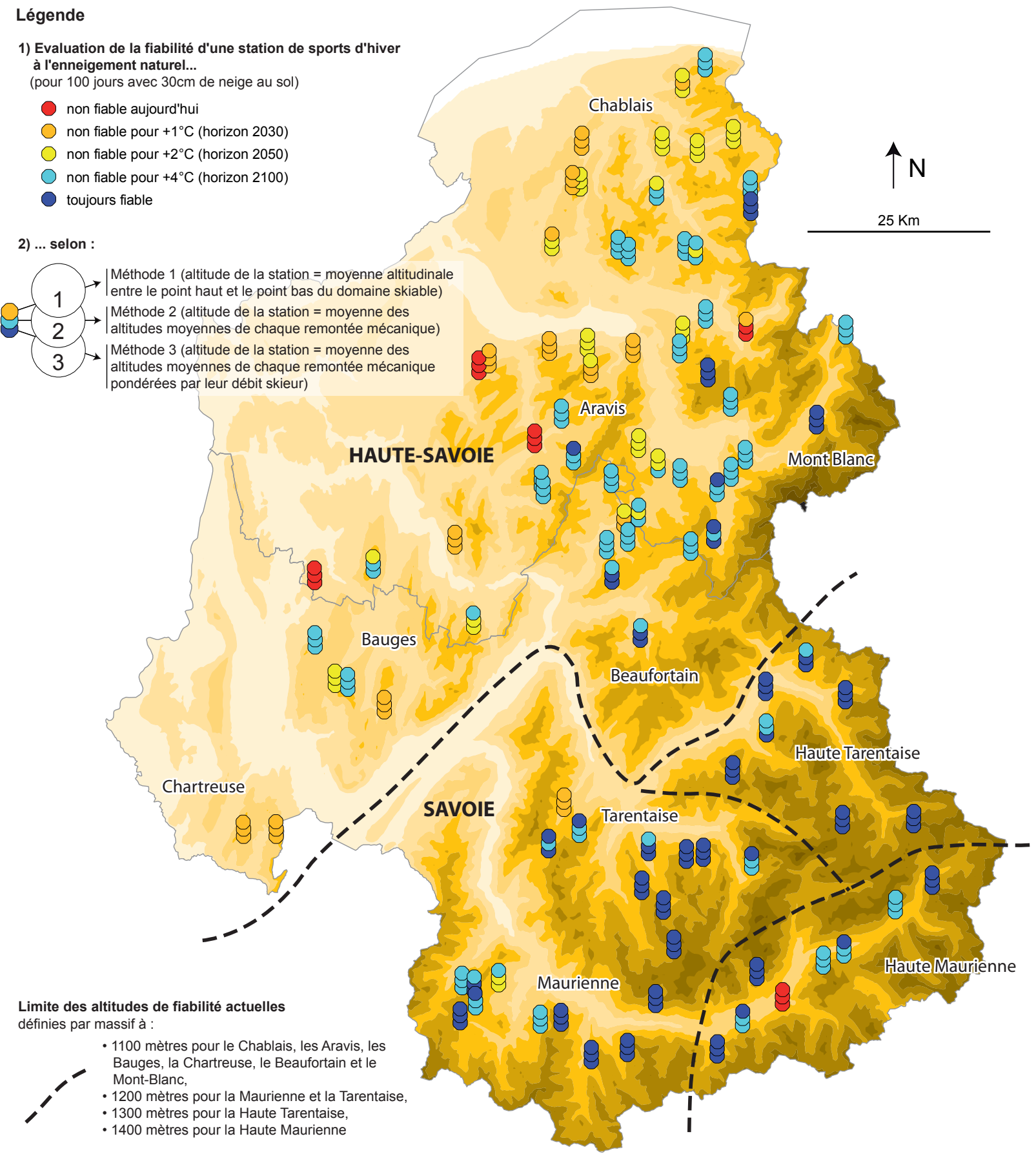

Figure 6 - Evaluation de la fiabilité de l'enneigement des domaines skiables de Savoie et Haute-Savoie. Chaque station est ici figurée par un ensemble de trois points. La couleur de chacun de ces points représente le résultat de l'évaluation de la fiabilité de la station selon les méthodes 1, 2 ou 3. 


\section{a - Résultats}

A la lecture de ces résultats, force est de constater une hétérogénéité des résultats selon la méthode de calcul utilisée. Une station peut ainsi être considérée à la fois comme fiable ou non fiable pour un même horizon selon l'altitude qui lui est affectée (méthode 1, 2 ou 3). Les conséquences du réchauffement climatique sur la ressource neige en station de sports d'hiver peuvent ainsi être appréhendées d'une façon différente selon les méthodologies conduites.

Par ailleurs, ces résultats nous montrent une grande diversité des situations intra et inter départementales. Ces différentes situations sont à relativiser selon le poids qu'occupe chaque site dans l'offre touristique. Les grands domaines d'altitude de Maurienne ou de Tarentaise par exemple, détenant une part importante de l'offre de ski alpin, semblent être relativement protégés. Les domaines skiables des massifs de moindre altitude au contraire, s'ils sont ici présentés comme les moins fiables du point de vue de l'enneigement naturel, ne représentent néanmoins qu'une faible part de l'offre touristique de ski alpin considérée dans sa globalité.

La synthèse des résultats, présentée à l'échelle départementale dans le tableau 4, montre les variabilités des calculs de fiabilité selon les différentes approches et les compare aux résultats de l'OCDE. Si les méthodes proposées donnent des résultats globalement homogènes, ceux-ci présentent une différence significative avec les résultats de l'OCDE. Cette différence peut s'expliquer par un nombre plus important de sites de faible altitude pris en compte dans notre analyse et par la définition d'altitudes de fiabilité différentes.

\section{b - Discussion}

A partir de cette méthodologie, nous voulions répondre aux principales limites de l'analyse proposée par l'OCDE. Si les méthodes utilisées ne remettent globalement pas en cause les résultats de l'OCDE, celles-ci permettent cependant une cartographie plus précise et argumentée de la fiabilité de l'enneigement des stations étudiées. En réalité, trois manques principaux à notre contribution perdurent. Dans un premier temps, les données météorologiques se sont révélées trop peu nombreuses pour permettre d'affiner une altitude de fiabilité de l'enneigement par massif. Ce manque conduit finalement à justifier a posteriori la limite globale des 1200 mètres de l'OCDE que nous voulions préciser. Cette altitude devrait donc être retravaillée par l'analyse d'un nombre plus important de stations météorologiques et de séries de données plus longues.

Ensuite, notre analyse reste focalisée sur le concept de limite de fiabilité de l'enneigement naturel définie par massif, sans prise en compte d'autres paramètres physiques locaux pouvant influencer l'enneigement : topographie des sites, exposition des versants, etc. En outre, la part d'incertitude relative aux conséquences

\begin{tabular}{|c|c|c|c|c|c|c|c|c|}
\hline \multicolumn{4}{|c|}{ Fiabilité } & \multirow{2}{*}{$\begin{array}{c}\text { non fiable } \\
\text { aujourd'hui } \\
0\end{array}$} & \multirow{2}{*}{$\begin{array}{c}\text { non fiable } \\
\text { pour }+1^{\circ} \mathrm{C} \\
(2030) \\
2\end{array}$} & \multirow{2}{*}{$\begin{array}{c}\text { non fiable } \\
\text { pour }+2^{\circ} \mathrm{C} \\
(2050) \\
4\end{array}$} & \multirow{2}{*}{$\begin{array}{c}\text { non fiable } \\
\text { pour }+4^{\circ} \mathrm{C} \\
(2100) \\
12\end{array}$} & \multirow{2}{*}{$\begin{array}{c}\text { toujours } \\
\text { fiable } \\
30\end{array}$} \\
\hline \multirow{16}{*}{ 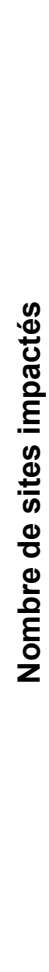 } & \multirow{8}{*}{ 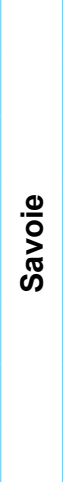 } & \multirow{2}{*}{\multicolumn{2}{|c|}{$\begin{array}{l}\text { d'après OCDE (2007) } \\
\text { [sur } 42 \text { sites analysés] }\end{array}$}} & & & & & \\
\hline & & & & $0 \%$ & $5 \%$ & $10 \%$ & $29 \%$ & $71 \%$ \\
\hline & & \multirow{6}{*}{$\begin{array}{l}\text { d'après les } \\
\text { altitudes } \\
\text { de fiabilité } \\
\text { définies par } \\
\text { massifs } \\
\text { [sur } 48 \text { sites } \\
\text { analysés] }\end{array}$} & \multirow{2}{*}{$\begin{array}{c}\text { méthode } \\
1\end{array}$} & 1 & 5 & 7 & 24 & 24 \\
\hline & & & & $2 \%$ & $10 \%$ & $15 \%$ & $50 \%$ & $50 \%$ \\
\hline & & & \multirow{2}{*}{$\begin{array}{c}\text { méthode } \\
2\end{array}$} & 1 & 6 & 8 & 25 & 23 \\
\hline & & & & $2 \%$ & $13 \%$ & $17 \%$ & $52 \%$ & $48 \%$ \\
\hline & & & \multirow{2}{*}{$\begin{array}{c}\text { méthode } \\
3\end{array}$} & 1 & 6 & 8 & 23 & 25 \\
\hline & & & & $2 \%$ & $13 \%$ & $17 \%$ & $48 \%$ & $52 \%$ \\
\hline & \multirow{8}{*}{ 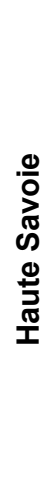 } & \multirow{2}{*}{\multicolumn{2}{|c|}{$\begin{array}{l}\text { d'après OCDE (2007) } \\
\text { [sur } 37 \text { sites analysés] }\end{array}$}} & 2 & 10 & 19 & 30 & 7 \\
\hline & & & & $5 \%$ & $27 \%$ & $51 \%$ & $81 \%$ & $19 \%$ \\
\hline & & \multirow{6}{*}{$\begin{array}{l}\text { d'après les } \\
\text { altitudes } \\
\text { de fiabilité } \\
\text { définies par } \\
\text { massifs } \\
\text { [sur } 47 \text { sites } \\
\text { analysés] }\end{array}$} & \multirow{2}{*}{$\begin{array}{c}\text { méthode } \\
1\end{array}$} & 3 & 11 & 23 & 41 & 6 \\
\hline & & & & $6 \%$ & $23 \%$ & $49 \%$ & $87 \%$ & $13 \%$ \\
\hline & & & \multirow{2}{*}{$\begin{array}{c}\text { méthode } \\
2\end{array}$} & 4 & 12 & 24 & 44 & 3 \\
\hline & & & & $9 \%$ & $26 \%$ & $51 \%$ & $94 \%$ & $6 \%$ \\
\hline & & & \multirow{2}{*}{$\begin{array}{c}\text { méthode } \\
3\end{array}$} & 4 & 11 & 21 & 43 & 4 \\
\hline & & & & $9 \%$ & $23 \%$ & $45 \%$ & $91 \%$ & $9 \%$ \\
\hline
\end{tabular}

Tableau 4 - Variabilités des calculs de fiabilité selon les différentes approches 
régionales du réchauffement climatique n'est ici pas prise en compte dans les variations de la limite de fiabilité en fonction des augmentations de température projetées.

Enfin, tant dans la méthodologie conduite - centrée sur l'aléa - que dans la représentation même des résultats, présentés sous forme cartographique, il est certain que notre contribution accentue a priori une lecture déterministe du risque lié au réchauffement climatique (Marcepoil et Boudières, 2008). Pour combler cette lacune, il parait donc nécessaire de compléter cette prospection du risque d'un déficit de neige par une approche centrée sur la vulnérabilité de ces terrains d'étude. Il s'agirait dans cette perspective d'analyser la prise en compte du réchauffement climatique par leurs acteurs et donc leur capacité à y faire face.

\section{Conclusion}

Evaluer la fiabilité des stations du point de vue de leur enneigement naturel dans un contexte de réchauffement climatique est un objectif difficile à tenir. Reprenant les grands principes des travaux réalisés sur le sujet par l'OCDE, notre contribution propose une réflexion méthodologique simple et appliquée à une échelle départementale. Celle-ci reste néanmoins à relativiser selon d'autres paramètres locaux : exposition des versants, topographie des sites, etc. En ce sens, c'est à une échelle encore plus fine qu'il faudrait pouvoir raisonner, celle de chaque station de sports d'hiver.

Par ailleurs, pour compléter notre évaluation du risque centrée sur l'aléa - en l'occurrence le risque d'un déficit récurrent de neige dans les prochaines années -, il conviendrait de s'intéresser également aux stratégies des stations de sports d'hiver pour y faire face. Pour Elsasser et Bürki (2002), les différentes stratégies employées sont :

- le maintien de l'activité ski par profilage des pistes, coopération (par exemple une assurance mutualisée contre les mauvaises saisons), production de neige de culture ou développement des domaines skiables à des altitudes plus élevées,

- le fatalisme soit en ne changeant rien, soit en arrêtant tout,

- l'aide aux secteurs en difficulté par des politiques de subvention,
- le choix d'une diversification de l'offre touristique proposée en stations. A ce sujet, si la diversification des activités touristiques est effectivement parfois envisagée, la difficulté réside cependant toujours à mettre en œuvre, à périmètre constant, une alternative aux retombées économiques aussi importantes que le produit ski.

La neige de culture est effectivement une des solutions largement employées aujourd'hui pour atténuer les effets de déficits de neige. Très concrètement, si la production de neige vise à préparer une sous-couche de neige à l'avant saison, garantissant l'ouverture des domaines skiables à date fixe, elle permet également de minimiser les pertes les années de faible enneigement en garantissant l'ouverture d'un domaine skiable minimal. Il s'agit ainsi de se prémunir des conséquences probables du réchauffement climatique sur la ressource neige.

La fiabilité de l'enneigement naturel, au regard des capacités d'adaptation des stations de sports d'hiver, n'est ainsi qu'un des éléments à prendre en considération dans un objectif de définition des vulnérabilités de ces territoires touristiques de montagne. Cela est d'autant plus vrai que les trajectoires d'évolution de l'offre et la demande touristique à long, voire à moyen termes, c'est-à-dire aux horizons 2030 à 2100 envisagés dans notre démarche, reste une inconnue.

\section{Remerciements}

Je remercie tout particulièrement la Direction Départementale de l'Equipement et de l'Agriculture de Savoie pour son accueil dans le cadre d'un monitorat en entreprise. Mes remerciements s'adressent également à la Direction Départementale de l'Equipement et de l'Agriculture de Haute-Savoie, la Direction Régionale de l'Environnement Rhône-Alpes et ODIT France ; ce travail n'aurait en effet pas pu être conduit sans les échanges régulièrement et collectivement entretenus avec l'ensemble de ces administrations. Merci enfin à Météo France pour les données météorologiques mises à ma disposition ainsi qu'à Christophe Chaix pour sa précieuse aide quand au traitement de celles-ci.

\section{BibliogRAPHIE}

Aspen Global Change Institute, 2006. Climate Change and Aspen : an assessment of impacts and potential responses. $178 \mathrm{p}$.

Berlioz F., 2008, Plan climat et tourisme. In Plan Climat Savoie 2020, le 18/11/2008, le Bourget du Lac.
Dawson J., 2008, Les stations d'Amérique du Nord face au changement climatique. In Colloque les entretiens de la Montagne, 10ème édition - l'économie touristique pilier du développement durable, le 06/11/2008, Chambéry.

Elsasser H., BÜRKI R., 2002, Climate change as a threat to tourism in the Alps. Climate Research, 20, 253-257. 
Etchevers P., Martin E., 2002. Impact d'un changement climatique sur le manteau neigeux et l'hydrologie des bassins versants de montagne. In: Colloque international : L'eau en Montagne - Gestion intégrée des Hauts Bassins Versants, le 05/09/2002, Megève.

FöHN P., 1990. Schnee und Lawinen. Schnee, Eis und Wasser der Alpen in einer wärmeren Atmosphäre. Internationale Fachtagung, Mitteilungen Versuchanstalt für Wasserbau, Hydrologie und Glaziologie der ETH Zürich, 108, 33-48.

Helion C., 2006. Transports par câbles et sports d'hiver : approche géographique de la dynamique territoriale du tourisme et des loisirs en espace montagnard. Collection EDYTEM, Cahier de Géographie, 4, 105-114.

LATERNSER M., SchneEbeli M., 2003. Long-term snow climate trends of the Swiss Alps, 1931-99, International journal of climatology, 23, 730-750.

LOUBIER J.-C., 2007. Changement climatique et domaines skiables : simulation en Savoie et Haute Savoie à l'horizon 2015. Mappemonde, 85, accès au 30/01/2008, http:// mappemonde.mgm.fr/num13/articles/art07103.html

Marcelpoil E., Boudieres V., 2008. Climate change and mountain touristic territories vulnerability : an endogenous reading. In Conference Managing Alpin Futur, le 01/04/2008, Innsbruck, Autriche.

MÉtÉo France, 2009. Point sur l'enneigement dans les massifs français au 5 février 2009, accès au 05/02/2009, http://france.meteofrance.com/france/actu/ actu?document_id $=20350 \&$ portlet_id $=42259$
Montagne Leader, 2008. Top 100 des opérateurs français de domaines skiables. Montagne Leader, 209, 45-56.

OCDE, 2007. Changements climatiques dans les alpes européennes Adapter le tourisme d'hiver et la gestion des risques naturels. Sous la direction de Shardul Agrawala, Editions OCDE, $136 \mathrm{p}$.

Odit France, 2006. Les chiffres clés du tourisme de montagne en France. $5^{\text {ème }}$ édition. ODIT France, Paris, 58 p.

Odit France, 2008. Les domaines skiables face aux aléas d'enneigement et le développement de la neige de culture, ODIT France, Paris, $13 \mathrm{p}$.

Prudent Richard G., 2008. Changements climatiques dans les Alpes : Impacts et risques naturels. Rapport Technique $\mathrm{N}^{\circ} 1$ de l'ONERC, Observatoire National sur les Effets du Réchauffement Climatique, $87 \mathrm{p}$.

Reynaud L., 2008, Le changement climatique et le futur du tourisme de montagne. In Salon mondial du tourisme de neige, le 27-29/03/2008, Andorre.

Tabeaud M., Delaporte B., 2005. Manteau et tourisme hivernal dans les alpes : Les savoyards et l'or blanc. Perceptionclimat.net, 8 pp., accès au 28/10/2008, http:// www.perceptionclimat.net/info.php?id=3 



\section{NEIGE et GLACE de MONTAGNE \\ Reconstitution, dynamique, pratiques}

\section{Sommaire}

Editorial

Introduction

\section{1 - Reconstitution}

Coutterand S. et al. - Le lobe glaciaire lyonnais au maximum würmien : glacier du Rhône ou/et glaciers savoyards ?

Ravanel L. et al. - Désenglacement du haut bassin versant du Vorz (massif de Belledonne, Isère), au Tardiglaciaire et à l'Holocène.

Rey P.-J. - Sociétés et fluctuations du climat dans les Alpes nord-occidentales au Néolithique moyen.

Le Roy et al. - Étude des fluctuations glaciaires du Petit Âge de Glace dans le Massif des Écrins : apports de la lichénometrie.

Kirkbride M.P. - Datation des moraines holocènes d'Islande par tephrochronologie : un état de l'art.

Le Roy et al. - La dendroglaciologie, ou l'apport de l'étude des cernes d'arbres pour la reconstitution des fluctuations glaciaires holocènes.

Rabatel A. - Évolution glaciaire dans les andes subtropicales chiliennes entre 1955 et 2007 : conséquences pour la ressource en eau.

Le Roy et al. - Un inventaire des aérophotographies du massif du Mont Blanc.

\section{2 - Dynamique}

Ravanel L. - Évolution géomorphologique de la haute montagne alpine dans le contexte actuel de réchauffement climatique.

Gruber S. - Le permafrost de haute montagne.

Deline P. et al. - L'Aiguille du Midi (massif du Mont Blanc) : un site remarquable pour l'étude du permafrost des parois d'altitude.

Saulnier G.-M. et al. - Un éléphant volant est-il un oiseau ? Perspectives pour l'observation hydrométéorologique des milieux de montagne.

Jobard S. - L'instrumentation du glacier du Baounet : quels apports pour la traçabilité des mesures environnementales ?

Moreau L. - L'exploration du cryokarst glaciaire et son intérêt scientifique pour l'étude du drainage des eaux de fonte.

Mazué R. et al. - Suivi de l'évolution de la couverture detritique d'un glacier noir par photo-comparaison : le glacier d'Estelette.

\section{3 - Pratiques}

Paccard P. - Réchauffement climatique et ressource neige en domaines skiables.

Gauchon C. - Les hivers sans neige et l'économie des sports d'hiver : un phénomène récurrent, une problématique toujours renouvelée.

Laslaz L. - L'exclusion des glaciers des zones centrales des Parcs nationaux de la Vanoise et des Écrins et leur équipement pour le ski d'été.

Cayla N. - Les sentiers d'interprétation glaciaire : des outils de valorisation différenciée des glaciers et de leur territoire.

Lambert R. - Cartozonage : de la carte au zonage du risque avalanche.

Moulin A. et al. - L'incertitude liée aux avalanches dans les Alpes du Nord : identifications et implications pour la gestion.

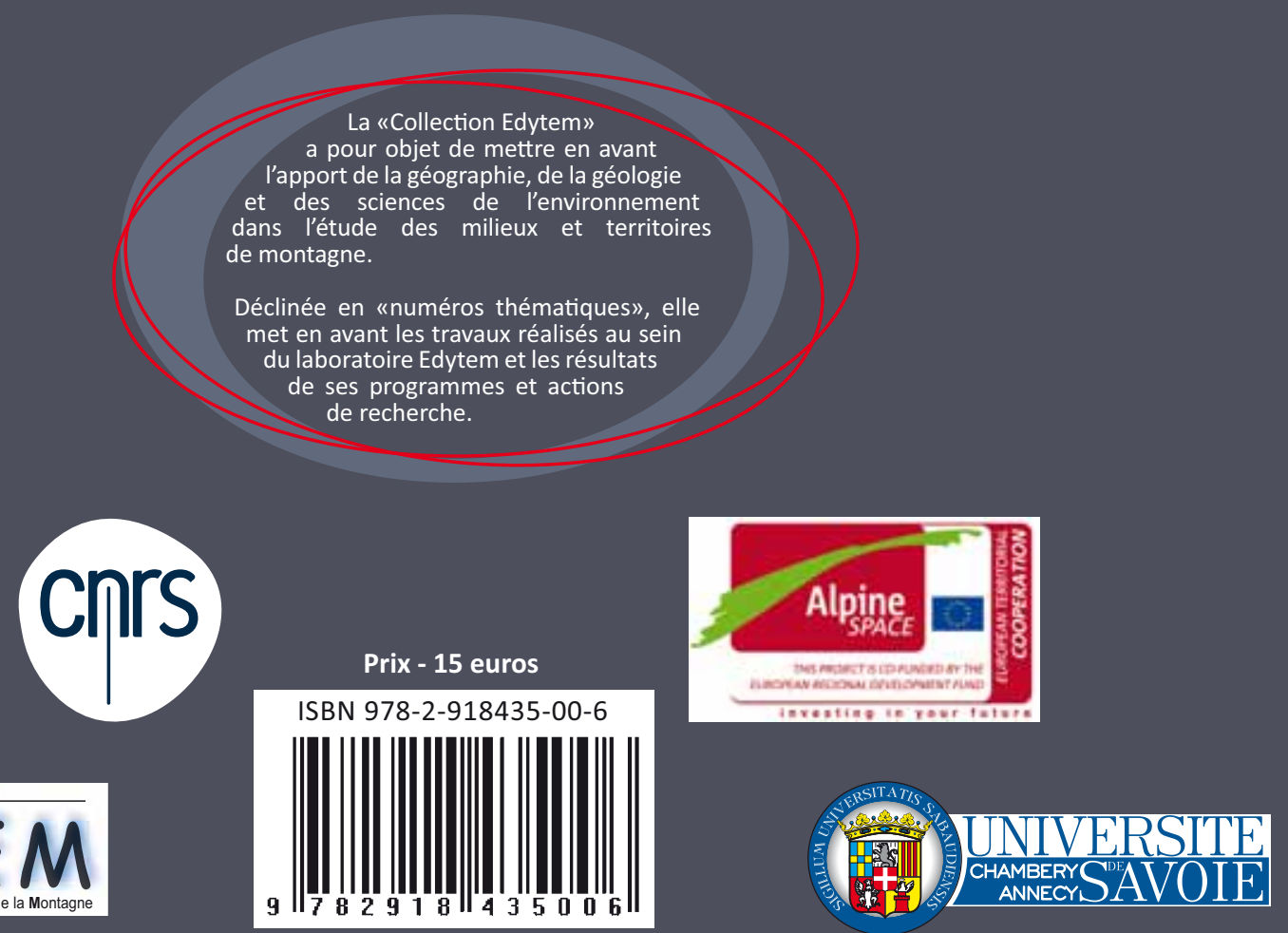

